\title{
Differential Electron Scattering Cross-Section at Low Electron Energies: The Influence of the Screening Parameter
}

\author{
Martin Čalkovský ${ }^{1}$, Milena Hugenschmidt ${ }^{1}$, Erich Müller ${ }^{1}$ and Dagmar Gerthsen ${ }^{1}$ \\ 1. Laboratory for Electron Microscopy, Karlsruhe Institute of Technology (KIT), Karlsruhe, Germany.
}

The interest in the electron microscopy at low electron energies is rising because knock-on damage can be avoided, and high contrast is obtained especially for weakly scattering materials [1]. Moreover, low energy scanning transmission electron microscopy (STEM) can be performed in a dual-beam focusedion beam/scanning electron microscope (FIB/SEM) equipped with a STEM detector at electron energies $\leq 30 \mathrm{keV}$ enabling preparation and direct investigation of electron transparent samples. Images obtained by high-angle annular dark field (HAADF) STEM imply material (Z-)dependent contrast which can be exploited to derive atomic numbers and also local specimen thicknesses. To extract quantitative information from HAADF STEM images, experimental images must be compared with simulations with defined input parameters which characterize specimen (material parameters, sample thickness) and imaging parameters (electron energy, collection angle range). Monte Carlo (MC) simulations are well established to calculate the interaction between electron and a target material and can be used to calculate HAADF STEM intensities I IAADF on a non-atomic level [2]. Previous work has shown that discrepancies exist between experimental and simulated $\mathrm{I}_{\mathrm{HAADF}}$ in some cases, which are mainly attributed to the differential scattering cross-sections used in MC simulation (DSCS) [3].

In this work we focus on the importance of the screening parameter in DSCS for MC simulations and on the contribution of the screening parameter to the $\mathrm{Z}$ dependence of IHAADF. We have studied 9 different materials with (average) atomic numbers between 3.5 and 74 using electron energies of $10 \mathrm{keV}, 20 \mathrm{keV}$, and $30 \mathrm{keV}$. Several screening parameters from literature were tested and failed to generally describe the experimental $\mathrm{I}_{\mathrm{HAADF}}$. Hence, adapted screening parameter were determined to obtain the best match between experimental I HAADF and MC simulations. Finally, the overall Z dependence of I HAADF was derived.

Samples with a well-known thickness profiles were obtained by FIB milling wedges from bulk materials (cf. Fig. 1a)). HAADF-STEM images were acquired and intensity line profiles were extracted starting from the thin wedge edge towards increasing specimen thickness (red dashed line in Fig. 1b)). For MC simulations the NISTMonte simulation package [4] with screened Rutherford DSCSs was used. Six different screening parameters [5,6] for screened Rutherford DSCSs were tested and simulated IHAADF were compared with measured data. In addition, the detection properties of the semiconductor STEM detector were taken into account.

Fig. 2a) shows an example for the comparison of the measured $\mathrm{I}_{\text {HAADF }}$ (blue line) as a function of the specimen thickness $t$ and $M C$ simulations for diamond-like carbon (DLC). I IAADF $(t)$ first increases, reaches a maximum and decreases if electrons are scattered in very large angles beyond the HAADF-STEM detector segment. It is obvious that simulated curves (here for Bishop, Nigam and Moliere screening) do not agree with the measured data. All investigated screening parameters [5,6] comprise a screening radius in form of $R \sim \mathrm{Z}^{-1 / 3}$, but none of them allows to consistently fit the measured

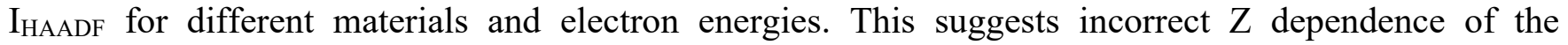
screening parameter, i.e., screening radius $R$. Hence, the screening radius was manually adjusted for 
each invested single-element material (DLC, $\mathrm{Si}, \mathrm{Ge}, \mathrm{Pd}, \mathrm{W}$ ) to obtain best possible fit. An example is shown in Fig. 2b), where the best fit for DLC was obtained with $R=0.58 a_{o}$ ( $a_{o}$ : Bohr radius) at 10, 20 and $30 \mathrm{keV}$. The determined screening radii for all invested single-element materials are plotted as a function of $\mathrm{Z}$ in Fig. 2c). The fit curve yields a new expression for the screening radius $R=1.28 \mathrm{Z}^{-0.44}$. The validity of this equation was tested by comparing experimental and simulated $\mathrm{I}_{\text {HAADF }}(\mathrm{t})$ of compounds $\left(\mathrm{ZnO}, \mathrm{MgO}, \mathrm{SrTiO}_{3}\right)$, which also yields good agreement. With the new screening parameter, the overall Z-dependence of I IAADF in low-energy HAADF STEM can be estimated by integration of the DSCS over the HAADF scattering angle range [6] and is found to be proportional to $Z^{1.58}$ [7].

\section{References:}

[1] M.F.G. Klein et al., J. Polym. Sci. B Polym. Phys. 50 (2012), p. 198.

[2] H. Demers et al., Scanning 33 (2011), p. 135.

[3] T. Volkenandt et al., Microsc. Microanal. 111 (2014) p.20.

[4] N.W.M. Ritchie, Surf. Interface Anal. 37 (2005), p. 1006.

[5] G. Moliere, Zeitschrift für Naturforschung A 2 (1947), p. 133.

[6] I. Kyriakou, J. Appl. Phys. 113 (2013), p. 084303.

[7] We are grateful for funding by the Deutsche Forschungsgemeinschaft (DFG).

a)
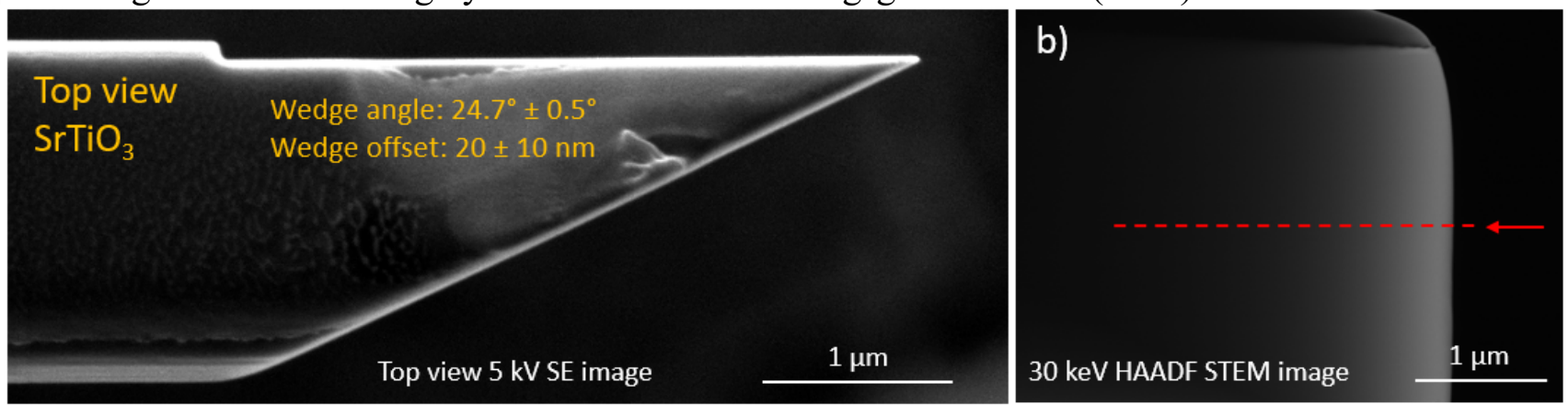

Figure 1. a) $5 \mathrm{keV}$ top-view secondary electron $\mathrm{SEM}$ image of the $\mathrm{SrTiO}_{3}$ wedge, enabling the determination of the wedge angle and thickness at the wedge edge. b) $30 \mathrm{keV}$ HAADF-STEM image of the $\mathrm{SrTiO}_{3}$ wedge in side perspective, where the thickness of the wedge increases from left to right.
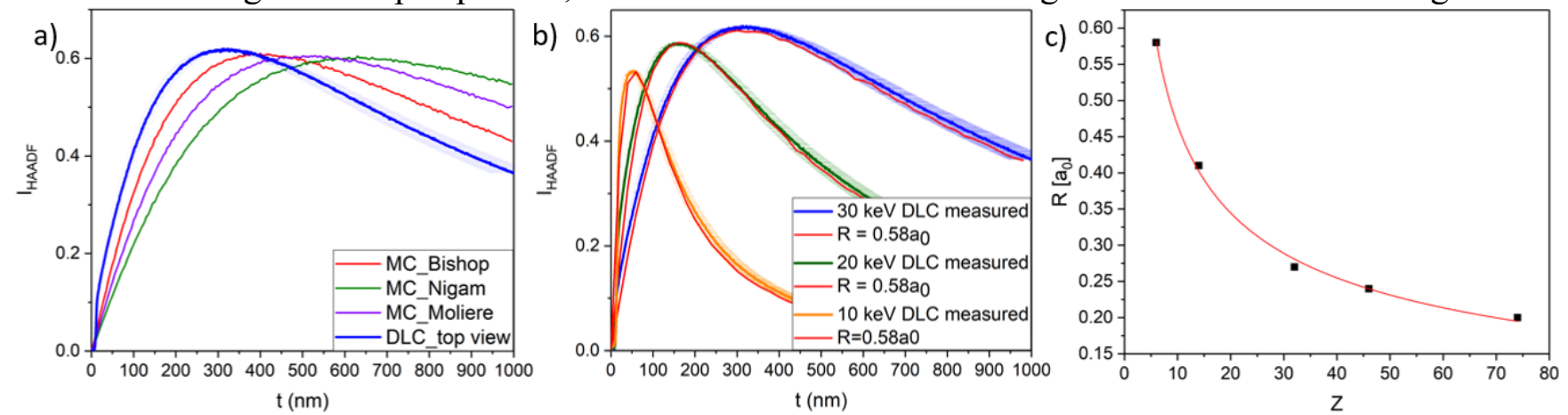

Figure 2. a) Measured $\mathrm{I}_{\mathrm{HAADF}}$ (normalized with the intensity of the incident electron beam) as a function of thickness and MC simulations with different screening parameters for the DLC wedge. b) Comparison of measured and simulated $\mathrm{IHAADF}_{\mathrm{H}}(\mathrm{t})$ with manually adjusted screening radius for DLC for 30,20 , and $10 \mathrm{keV}$. c) Screening radii for all investigated single-element materials plotted as a function of $Z$ and fit by a power-law function. 